\title{
NON-SMOOTH GEODESIC FLOWS AND \\ CLASSICAL MECHANICS
}

\section{J.E. Marsden}

As is well known, there is an intimate connection between geodesic flows and Hamiltonian systems. In fact, if $g$ is a Riemannian, or pseudo-Riemannian metric on a manifold $M$ (we think of $M$ as q-space or the configuration space), we may define a smooth function $T_{g}$ on the cotangent bundle $T * M$ (q-p-space, or the phase space). This function is the kinetic energy of $q$, and locally is given by

$$
T_{g}(q, p)=\frac{1}{2} \underset{i, j}{\Sigma} g^{i j}(q) p_{i} p_{j}
$$

where $q=\left(q^{1}, \ldots, q\right)$ and $p=\left(p_{1}, \ldots, p_{n}\right)$ and $g$ has components $g^{i j}$. Using $T_{g}$ as a Hamiltonian function, the associated flow (that is, the global solution of Hamilton's equations) is exactly the geodesic flow; geodesics are obtained by projection to $M$.

Conversely, Hamiltonian motion in a potential $\mathrm{V}$ and metric $\mathrm{g}$, that is, $\mathrm{H}=\mathrm{T}_{\mathrm{g}}+\mathrm{V}$, may be thought of as geodesic motion using the metric $(e-v) g$ if $e>V(q)$. This new metric is called the Jacobi metric.

Traditionally, the theory of classical mechanics and Riemannian geometry always assumes $\mathrm{g}$ and $\mathrm{V}$ are smooth functions. However, the most elementary examples in fact are not smooth; see below. One of the main reasons for the smoothness as sumption was to guarantee existence of the flow (geodesics). This objection has now been removed.

The purpose of this note is to explain in an expository fashion what changes are necessary in the above theory to cover the non-smooth case. This new situation is quite different, although some interesting observations can be made.

The main theorem in the non-smooth theory is that if $H=T g+V$ is singular only on a set of measure zero ( $H$ is a distribution in general). then there exists a measurable flow $F_{t}: T * M \rightarrow T * M$ defined almost

everywhere which can be assigned to $H$. This flow is, in fact, the pointwise limit of smooth flows corresponding to smooth approximations of $\mathrm{H}$. We shall denote the approximate quantities with a superscript $k$. Thus

$$
F_{t}^{k}(m) \rightarrow F_{t}(m)
$$


for almost all $t, m$. Of course the smooth flows are obtained in the usual manner. For the technical machinery needed to carry out this program, see [2].

This theorem is an important result. It gives us, for the first time, a systematic method for investigating geodesics which are allowed to have sharp corners.

The geodesic flow has a fundamental property in common with the smooth case. That is, the length of the tangent to a geodesic (wherever the geodesic has onel, is of constant length. This corresponds exactly to conservation of energy, and holds even when we cross over singularities. To clarify this and other properties below, we consider two examples:

1. For $q$ space take $\mathbf{R}^{2}$ the Euclidean plane so that $q-p$ space is $R^{4}$. Employ the Euclidean metric for $g$. As a potential, let $V$ be a delta "function" spread uniformly along the $q^{2}$-axis. Roughly, $V\left(q^{1}, q^{2}\right)=\delta\left(q^{1}\right)$. The corresponding motion in $q-s p a c e$ (geodesics of the Jacobi "metric") is just the free motion of particles reflecting (elastically) from a "wall" along the $\mathrm{q}^{2}$ axis. The flow is undefined along the $q^{2}$-axis. Note that the flow is continuous in $t$ in the configuration component $q$, but not in the $p$ component. This is a general phenomena proven below.

2. Again take $M=R^{2}$ and $g$ the Euclidean metric. This time define $v$ by

$$
v\left(q^{1}, q^{2}\right)=\left\{\begin{array}{l}
0 \text { if } q^{1}<0 \\
1 \text { if } q^{1} \geq 0
\end{array}\right.
$$

The flow on $M$ is now the refraction of particles according to Snell's law as they cross the interface along the $q^{2}$-axis.

These two examples illustrate how the variational theory of geodesics can go astray. In the first example the method essentially fails. That is, a path undergoing reflection does not extremize, more specifically minimize, the Lagrangian or Jacobi metric integrated over the path. The Lagrangian is just $L=T_{g}-V$. For example, by pulling the reflection point slightly away from the wall, decreases the quantity

$$
\int_{0}^{1} L(q(t), P(t)) d t
$$


while the opposite motion increases it. On the other hand, the second example, as is well known does have the extremal property. What is the difference?

To answer this question, we make a definition which distinguishes the two cases. We say a flow $F_{t}$ of $H=T_{g}+V$ is regular if and only if $T_{g}$ and $V$ are (locally) bounded functions and each two points $q$, $q$ can be joined by a smooth geodesic $c^{k}(t)$ from the approximating system $H^{k}=T_{g}^{k}+V^{k}$ which (i) minimizes its Langrangian, and (ii) $c^{k}(t)$ converge as $k \rightarrow \infty$ to the geodesic of $H$. We also assume $L^{k} \rightarrow L$ boundedly.

Clearly, example one is not regular, but the second is. Notice that although the flows $F_{t}{ }^{k}$ converge, this does not imply that geodesics with fixed endpoints converge. In fact, in example one, geodesics joining points on opposite sides of the wall diverge, and in the limit two such points cannot be joined by a geodesic. Here is an important difference with the smooth case.

The basic fact is that in the regular case, we always retain the extremal property. This is quite easy to see. In fact, if $c^{k}(t)$ is an approximating geodesic and $\hat{c}(t)$ is any other curve.

$$
\int L^{k}\left(c^{k}(t)\right) d t \leq \int L^{k}(\hat{c}(t)) d t ;
$$

letting $k \rightarrow \infty$ and employing the dominated convergence theorem gives

$$
\int L(c(t)) d t \leq \int L(\hat{c}(t)) d t .
$$

The curve $c(t)$ and $L$ are not differentiable, but this still makes sense. although the usual variational techniques fail. Another distinction between the examples is that we can define curvature in the second (it is a distribution), but not in the first. Again see [2] for the tools needed.

Intuition developed in classical mechanics and Riemannian geometry suggests that the paths in configuration space (geodesics) should always be continuous, although the momentum $p$ may be discontinuous. (The paths need not be continuous with respect to the initial conditions.) This is in fact true and is quite easy to see in this framework. We suppose $H=T_{g}+V$ where $g^{i j}$ are (locally) bounded functions and $V$ may be singular. (Both examples satisfy this.) From the equations for the approximating systems we have

$$
q^{i}(t)^{k}=\sum_{j} \int_{0}^{t} g^{i j}\left(q(s)^{k}\right)^{k} p_{j}(s)^{k} d s
$$


where $i, j$ refers to coordinates, Letting $k-\infty$ gives, by dominated convergence,

$$
q^{i}(t)=\Sigma \int_{j}^{t} g^{i j}(q(s)) p_{j}(s) d s .
$$

Therefore, $q(t)$ is continuous in $t$, as to be shown.

Further applications of these ideas may be found in [2]. The smooth case is done in [1] and background for geodesics is contained in [3].

\section{REFERENCES}

1. R. Abraham and J. Marsden, Foundations of mechanics. (Benjamin, N. Y., 1967).

2. J. Marsden, Generalized Hamiltonian mechanics. Arch. for Rat. Mech. and Analysis 28, 5 (1968) 323-361.

3. S. Helgas on, Differential geometry and symmetric spaces. (Academic Press, N.Y., 1962).

Princeton University 